\title{
Determination of Hormone Receptors, Human Epidermal Growth Factor Receptor 2 and Ki67 Status in Invasive Breast Carcinoma: A Concordance Study between Immunohistochemistry, Fluorescence in Situ Hybridization, and GeneXpert® Breast Cancer STRAT4 Assay
}

rajaa elaje ( $\nabla$ elaje.rajaa@gmail.com)

University of Hassan II Casablanca Faculty of Science Ain Chock: Universite Hassan II de Casablanca Faculte des Sciences Ain Chock https://orcid.org/0000-0002-1749-7178

Abdellah Naya

University of Hassan II Casablanca Faculty of Science Ain Chock: Universite Hassan II de Casablanca Faculte des Sciences Ain Chock Ayoub KHOAJA

CHUN Ibn Rochd: Centre Hospitalier Universitaire Ibn Rochd

Younes Zaid

University of Hassan II Casablanca Faculty of Science Ain Chock: Universite Hassan II de Casablanca Faculte des Sciences Ain Chock Mounia Oudghiri

University of Hassan II Casablanca Faculty of Science Ain Chock: Universite Hassan II de Casablanca Faculte des Sciences Ain Chock

Jodi Weidler

Cepheid

Mehdi Karkouri

CHUN Ibn Rochd: Centre Hospitalier Universitaire Ibn Rochd

\section{Research article}

Keywords: Breast cancer, hormone receptors, Human Epidermal Growth Factor Receptor 2, Ki-67 immunohistochemistry, FISH, Xpert STRAT4, GeneXpert

Posted Date: November 23rd, 2021

DOI: https://doi.org/10.21203/rs.3.rs-1044271/v1

License: @ (i) This work is licensed under a Creative Commons Attribution 4.0 International License. Read Full License 


\section{Abstract}

\section{Background}

The accurate assessment of hormone receptors (ER and PR), HER2 and Ki-67 proliferative index provides meaningful information about breast cancer prognosis and prediction of therapy response. Immunohistochemistry, the most common method for evaluating these prognostic biomarkers, can be impacted by numerous variabilities due to pre-analytical/analytical factors and subjective interpretation by pathologists. The Xpert ${ }^{\circledR}$ Breast Cancer STRAT4, a RT-qPCR based system, can be used to classify breast invasive carcinomas based on the assessment of these 4 biomarkers. In this study, we investigated the accuracy of RT-qPCR based mRNA expression levels in a closed, singleuse cartridge, automated system compared with the current gold standard, immunohistochemistry (IHC), and fluorescent in situ hybridization (FISH) for HER2 equivocal cases.

\section{Methods}

We evaluated ESR1, PGR, ERBB2 and MKi67 mRNA expression by Xpert Breast Cancer STRAT4 and ER, PR, HER2 and Ki67 by IHC (FISH for HER2 IHC 2+) in 200 formalin-fixed paraffin-embedded (FFPE) tissue blocks with invasive breast cancer, collected from the Pathology Department of Casablanca Ibn Rochd University Hospital.

\section{Results}

Concordance between Xpert @ Breast Cancer STRAT4 and IHC was 93.5\% for ER, 83.51\% for PR, 95\% for HER2 (92\% for IHC+FISH), and $81.20 \%$ for Ki67 (excluding intermediate IHC Staining $10 \leq \% \mathrm{HC}<20)$. The simple Kappa coefficient was, for ER, $0.830(\mathrm{P}<0,0001)$, $0.565(\mathrm{P}$ $<0,0001)$ for PR, $0.838(P<0,0001)$ for HER2-IHC, $0.771(P<0,0001)$ for HER2 IHC+FISH and, for, Ki67, $0.458(P<0,0001)$.

\section{Conclusions}

We demonstrated globally a high concordance between centrally assessed IHC, IHC+FISH and mRNA measurements of ER/ESR1 and HER2/ERBB2, and a moderate agreement between PR/PGR and Ki67/MKi67. These findings provide an additional, objective, and quantitative assessment of tumor receptor status in breast cancer.

\section{Background}

Breast cancer remains the most common malignancy in women worldwide, contributing to $25.4 \%$ of the total of new cases diagnosed in 2018 [1]. In Europe, roughly 494,000 new patients are diagnosed each year and an estimated 143,000 women will die of their disease [2]. In Morocco, approximately 11,000 new cases were diagnosed in 2020 [3], making it, by far, the most common malignancy in the country. Thereby, breast cancer constitutes the most common cause of cancer mortality among women in Morocco [3].

Breast cancer mortality has declined by $34 \%$, in the last 30 years, mainly due to improved treatment and early detection [4]. Contemporary assessment of estrogen receptors (ER) and progesterone receptors (PgR) protein expression, generally by immunohistochemistry, provides useful prognostic information for breast cancer patient outcomes and predicts response to endocrine therapy [5-8].

Among acquired alterations that have now been identified in human breast cancers, HER-2/neu gene amplification has received a great deal of attention in the last 20 years. Measurement of protein overexpression or gene amplification of Human Epidermal Growth Factor Receptor 2 (HER2 or ERBB2) conveys a potential clinical utility as a prognostic marker, but more importantly, as a predictor of responsiveness to trastuzumab or other HER2-targeted therapies $[9,10]$.

Many retrospective studies of breast cancer patients have broadly demonstrated the important prognostic value of the marker of proliferation Ki67 (MKi67). Potential uses comprise prognosis, prediction of response to chemotherapy or endocrine therapy, estimation of residual risk in patients on standard therapy and as a dynamic biomarker of treatment efficacy in samples taken before, during, and after neoadjuvant therapy $[10,11]$.

Accordingly, the European Society for Medical Oncology (ESMO) treatment guidelines recommend that all primary breast carcinomas should be tested for estrogen receptor (ER), progesterone receptor (PgR), HER2/ERBB2 and Ki-67 at the time of diagnosis[12-14].

In breast cancer, immunohistochemistry (IHC) on formalin-fixed paraffin-embedded (FFPE) is the gold standard for the assessment of hormonal receptor status, HER2 and Ki67[5, 10, 11]. Fluorescence in situ hybridization (FISH) is systematically used to clarify HER2 immunohistochemical results when HER2 score is equivocal (2+), while, a number of institutions routinely use FISH for initial HER2 evaluation status in all patients[9, 15]. 
In spite of long-term use, immunohistochemical assays have not been adequately standardized across labs and IHC and FISH results can be impacted by pre-analytical or analytical limitations, including tissue fixation, choice of antibodies, use of manual vs computer assisted scoring methods, and interpretation of results in assay performance, all of which can significantly affect the accuracy and reproducibility of results for these four biomarkers $[10,12,16,17]$.

The Xpert® Breast Cancer STRAT4 test is a CE-IVD* test (*In vitro diagnostic medical device. May not be available in all countries. Not available in the U.S.) that offers a semi-quantitative assay with qualitative cut-off values for Estrogen Receptor (ESR1), Progesterone Receptor (PGR), HER2/ERBB2, and Marker of Proliferation Ki-67 (MKi67) mRNAs isolated from FFPE invasive breast cancer samples[16, 17].

Xpert ${ }^{\circledR}$ Breast Cancer STRAT4 measures target (ESR1, PGR, ERBB2, and MKi67) and reference gene (CYFIP1) mRNAs isolated from FFPE breast cancer tissue in a self-contained cartridge using the Cepheid $\AA$ GeneXpert ${ }^{\circledR}(G X)$ System which automates and integrates the incartridge sample processing, including RNA isolation, amplification, and detection of the target sequences in FFPE samples using real-time reverse transcriptase, polymerase chain reaction assays (RT-PCR) [16, 18].

Current data suggest that Xpert® Breast Cancer STRAT4 is very reproducible and has a high degree of concordance with IHC (and HER2 FISH) results[16-24].

\section{Methods}

Two hundred blocks of FFPE tissue specimens aged $\leq 5$ years archived in the Pathology Department of Casablanca Ibn Rochd University Hospital were included in our study. The histopathology of all samples remaining in the blocks was reviewed, and only specimens still containing invasive breast carcinoma cells were included in the study. This analysis enrolled retrospectively de-identified FFPE tissue sections obtained from core biopsies or surgical specimens, from a selection of patients with invasive breast cancer whose tumor samples were collected and routinely evaluated for breast cancer biomarkers (ER, PR, HER2 and Ki67) according to the standard of care (SOC) IHC and/or FISH assays at the Pathology laboratory. The immunohistochemical status (IHC) was sought on $4 \mu \mathrm{m}$ tissue sections treated and incubated with the antibodies ER : FLEX Monoclonal Rabbit Anti-Human Estrogen Receptor a Clone EP1 Ready-to-Use; PR : FLEX Monoclonal Mouse Anti-Human Progesterone Receptor Clone PgR 636 Ready-to-Use and Ki67 : FLEX Monoclonal Mouse Anti-Human Ki-67 Antigen Clone MIB-1 Ready-to-Use, according to the Dako protocol on the Autostainer Link 48 IHC platform. HER2 status is first assessed by IHC, using the antibody Ventana Pathway Anti-HER-2/neu (4B5) Rabbit Monoclonal Primary Antibody on a Ventana GX automated platform. Tumors were classified as ER positive or PR positive when $\geq 1 \%$ invasive tumor cells showed definite nuclear staining, irrespective of staining intensity. A tumor was considered to be HER2 positive if an IHC score equal to 3+ was found and HER2 negative if a score of 0 or $1+$ was observed (ASCO/CAP guidelines)[25]. HER2 Equivocal (IHC 2+) results were subsequently tested by FISH with manual technique using the probes (HER2 IQFISH pharmDx) to confirm final HER2 status.

The patient tumors selected for our study represent the various breast cancer subtypes as determined through surrogate IHC subtyping by the routine assays performed at our laboratory, as follows:

-25 triple negative (ER negative, PR negative and HER2 negative)

-25 HER2+ (Hormone receptor (HR) negative / HER2 +)

-100 Luminal A (HR positive / Ki67 < $20 \%$ )

-25 Luminal B (HR positive / Ki67 $\geq 20 \%$ )

-25 HER2 IHC 2+ (8 cases HER2 FISH positive and 17 HER2 FISH negative using current ASCO/CAP HER2 guidelines)[26].

The mRNA levels of ESR1, PGR, ERBB2 (HER2), and MKi67 were assessed by quantitative gene expression readouts using the Xpert® Breast Cancer STRAT4 assay. Breast Cancer FFPE tissue samples were prepared for the assay as tissue scrolls (10 $\mu \mathrm{m}$ thickness) and placed into a tube. FFPE samples were first treated with the recommended volumes of FFPE lysis reagent ( $1.2 \mathrm{ml})$ and proteinase $\mathrm{K}(20 \mu \mathrm{L})$ provided by the Xpert ${ }^{\circledR}$ FFPE Lysis Kit (CE-IVD*) prior to use in Xpert ${ }^{\circledR}$ Breast Cancer STRAT4. The solution was then incubated in a heat block at $80^{\circ} \mathrm{C}$ for 30 minutes. Then $1.2 \mathrm{ml}$ of $\geq 95 \%$ Ethanol was mixed with the sample.

Once the tissue lysate is prepared, a $520 \mu \mathrm{L}$ aliquot was placed into the appropriate sample chamber in the Xpert® Breast Cancer STRAT4 cartridge. The testing cartridge was inserted into a module of a GeneXpert® System for processing where nucleic acid purification, amplification, and real-time detection are all fully automated and completely integrated by the system. The final results of STRAT4 testing are available in approximately 70 minutes after starting the test.

Page 3/15 


\section{Statistical analysis}

Statistical analysis was done in GraphPad Prism Software. For each of the four biomarkers studied, agreement measurements between Xpert ${ }^{\circledR}$ Breast Cancer STRAT4 and IHC and/or FISH, which were considered as the reference methods, were based on contingency table analysis and included overall concordance (overall percent agreement), positive percent agreement (sensitivity) defined as the number of samples classified positive by both IHC and Xpert® Breast Cancer STRAT4 divided by the number of positive samples using immunohistochemistry, negative percent agreement (specificity), and Cohen's $\mathrm{k}$ coefficient scores. The Kappa ( $\mathrm{K}$ ) statistic numeric values are categorized into slight agreement $(\leq 0.2)$, fair agreement (between 0.21 and 0.40 ), moderate agreement (between 0.21 and 0.40 ), substantial agreement (between 0.61 and 0.80 ) and almost perfect agreement (between 0.81 and 1.00). All measurements were associated with $95 \%$ confidence intervals $(95 \% \mathrm{Cl})$, compared using Fisher's exact test and considered significant for $\mathrm{P}<0.05[27]$.

\section{Results}

To assess the concordance between the Xpert $\circledast$ Breast Cancer STRAT4 and IHC+HER2 FISH methods, we used a cohort of 200 specimens of formalin-fixed paraffin-embedded invasive breast carcinomas diagnosed at the Pathology Department of Casablanca Ibn Rochd University Hospital between 2016 and 2020.

For each sample, we evaluated mRNA results by Xpert® Breast Cancer STRAT4 and compared them to the results obtained by the already routinely performed IHC+HER2 FISH .

The overall concordance rate between Xpert@ Breast Cancer STRAT4 ESR1 mRNA results and ER protein IHC results was 93.50\% (sensitivity $=97.26 \%$; specificity $=83.33 \%$; PPV $=94.04 \%$; NPV $=91.84 \%$, using either the IHC cut-off of $\geq 1 \%$ as recommended by ASCO-CAP [12] or $\geq 10 \%$ immunostaining level for positivity, and using a pre-defined delta Ct cut-off ( $\mathrm{dCt} \geq-1$ ) for ESR1-positivity by Xpert $\circledast$ Breast Cancer STRAT4 based on prior concordance studies. Only $2 \%$ of immunohistochemistry-ER-positive samples were classified negative using STRAT4, whereas $4.5 \%$ of immunohistochemistry-ER-negative samples showed a positive Xpert $\circledast$ Breast Cancer STRAT4 ER status (Fig. 1).

The Cohen's k coefficient score was equal to 0.830 ( $95 \%$ confidence interval: From 0.741 to 0.919 ).

Concordance between Xpert ${ }^{\circledR}$ Breast Cancer STRAT4 PGR and PR IHC results using an IHC cut-off of $\geq 1 \%$ as recommended by ASCO-CAP [12] was $83.51 \%$ (sensitivity $=96.3 \%$; specificity $=54.24 \%$; PPV $=82.80 \%$; NPV $=86.49 \%$ ) using PGR dCt cutoff of -3.5 . Only $2.5 \%$ cases of IHC PR-positive samples were classified negative by RT-qPCR, while 14\% of IHC PR-negative cases showed a positive Xpert $\circledast$ Breast Cancer STRAT4 PGR status based on current Xpert ${ }^{\circledR}$ Breast Cancer STRAT4 cutoffs. The statistical kappa value is around 0.565 ( $95 \%$ confidence interval: From 0.435 to 0.694 ). By using the IHC cut-off of $\geq 10 \%$ to determine PR-positive status, the overall concordance between both methods was $77.32 \%$ (sensitivity $=99.13 \%$; specificity $=45.57 \% ; P P V=72.61 \% ; N P V=97.30 \%$ ). In this case, only $0.5 \%$ of the samples were classified PR-positive by IHC and negative by Xpert ${ }^{\circledR}$ Breast Cancer STRAT4, whereas $22 \%$ of the samples of IHC PR-negative became Xpert@ Breast Cancer STRAT4 PGR-positive (Fig. 2).

The Cohen's K coefficient score was equal to 0.488 ( $95 \%$ confidence interval: From 0.372 to 0.603 ). Six cases with "indeterminate" Xpert ${ }^{\circledR}$ Breast Cancer STRAT4 PGR results were excluded from this analysis.

The overall concordance rate between Xpert $\circledast$ Breast Cancer Xpert ${ }^{\circledR}$ Breast Cancer STRAT4 ERBB2 mRNA and HER2 protein IHC results was approximately $95 \%$ (sensitivity $=90.91 \%$; specificity $=95.77 \% ;$ PPV $=83.33 \% ;$ NPV $=97.84 \%$ ), excluding equivocal cases (HER2 score $=2+$ ). The percentage of discordant cases classified by IHC as HER2- positive and Xpert $\circledast$ Breast Cancer STRAT4 ERBB2- negative was only $1.7 \%$, whereas 3.5\% of IHC HER2-negative cases were Xpert® Breast Cancer STRAT4 ERBB2- positive (Fig. 3).

In equivocal cases (IHC 2+), Xpert® Breast Cancer STRAT4 and HER2 FISH concordance was $68 \%$ (sensitivity $=87.50 \%$; specificity $=58.82 \%$ ; PPV $=50 \% ;$ NPV $=90.91 \%$ ). Solely $4 \%$ of FISH HER2-positive samples were classified negative using Xpert $\circledast$ Breast Cancer STRAT4 whilst $28 \%$ of FISH HER2-negative samples were classified positive using Xpert® Breast Cancer STRAT4 (Fig. 4).

The concordance rate between Xpert $\circledast$ Breast Cancer STRAT4 and IHC+HER2 FISH, including all samples was $92 \%$ (sensitivity $=92.50 \%$; specificity = 91.88\%; PPV = 74\%; NPV = 97.33\%) (Fig. 5).

The concordance rate obtained when the population was stratified first by ER status was $89.04 \%$ for HER2 ER+ and 100\% for HER2 ER-, including all cases.

The Cohen's K coefficient was equal to 0.838 (95\% confidence interval: From 0.735 to 0.940 ) when comparing Xpert $\circledast$ Breast Cancer STRAT4 ERBB2 dCt results to HER2 IHC results, excluding equivocal cases (HER2 IHC2+). The Cohen's $\mathrm{K}$ coefficient was equal to $0.387(95 \%$ 
confidence interval: From 0.073 to 0.700 ) when we analyzed IHC 2+ cases with Xpert® Breast Cancer STRAT4 ERBB2 dCt and FISH HER2 results. For determination of HER2 status taking into account both reference methods (IHC and FISH) as recommended by ASCO/CAP Guidelines [28] the Kappa coefficient is equal to 0.771 (95\% confidence interval: From 0.666 to 0.877 ). Considering the ER status stratified subset only for the comparison of Xpert® Breast Cancer STRAT4 ERBB2 dCt and HER2 results by IHC+HER2 FISH, the statistical Kappa was 0.521 ( $95 \%$ confidence interval: From 0.320 to 0.722 ) for ER-positive subset and 1.00 (95\% confidence interval: From 1.000 to 1.000 ) for ERnegative subset.

Last, we examined the Xpert® Breast Cancer STRAT4 MKi67 dCt values and Ki67 results by using Ki67 IHC cutoff of $10 \%$ and $20 \%$ to discriminate "high proliferation rate" from "low proliferation rate", while we used an intermediate zone (equivocal results) between 10 and 20\% for the MKi67 d Ct distribution. We excluded sixteen cases with « Indeterminate » Xpert® Breast Cancer STRAT4 MKi67 status from this study.

The overall concordance between Xpert ${ }^{\circledR}$ Breast Cancer STRAT4 MKi67 and Ki67 IHC considering positive as $\geq 20 \%$ was $67.39 \%$ (sensitivity $=96.84 \%$; specificity $=95.96 \%$; PPV $=61.74 \%$; NPV = 91.43\%). Only $1.6 \%$ of immunohistochemistry Ki67-positive samples were classified negative using Xpert ${ }^{\circledR}$ Breast Cancer STRAT4, while 31\% of immunohistochemistry Ki67-negative samples showed a positive Xpert ${ }^{\circledR}$ Breast Cancer STRAT4 status. When we considered $>10 \%$ cutoff, the overall agreement was $66.85 \%$ (sensitivity $=94 \%$; specificity $=34.52 \%$; PPV = 63.09\%; NPV = 82.86\%). Discordant Ki67 cases consisted of IHC-positive and Xpert® Breast Cancer STRAT4 -negative (3\%) and IHC-negative Xpert ${ }^{\circledR}$ Breast Cancer STRAT4 positive (30\%). When we excluded samples with IHC staining in the $10 \leq \% \mathrm{IHC}<20 \%$ range, the overall agreement was $81.2 \%$ (sensitivity $=96.84 \%$; specificity $=42.11 \%$; PPV $=80.70 \%$; NPV $=84.21 \%$ ). We noted here only $2 \%$ of discordant cases IHC-positive/ Xpert ${ }^{\circledR}$ Breast Cancer STRAT4 negative and 16.5\% of IHC-negative/ Xpert® Breast Cancer STRAT4 /positive (Fig. 6).

The MKi67/Ki67 Cohen's K coefficient is equal to 0.334 (95\% confidence interval: 0.224 to 0.445 ) using $20 \%$ Ki67 IHC Cutoff, Kappa = 0.299 (95\% confidence interval: 0.182 to 0.417 ) using $10 \% \mathrm{Ki} 67 \mathrm{IHC}$ Cutoff. When we excluded equivocal cases, the Kappa is equal to 0.458 (95\% confidence interval: From 0.289 to 0.628 ) (Table 1 ). 
Table 1

Comparison of protein status for ER, PR, HER2, and Ki67 and mRNA expression for ESR1, PGR, ERBB2 and MKi67 between Immunohistochemistry «IHC », Fluorescence in situ hybridization « FISH » and RT-qPCR « Xpert® Breast Cancer STRAT4 test*».

\begin{tabular}{|c|c|c|c|c|c|c|c|c|c|c|}
\hline Analyte & Reference & Total & $\begin{array}{l}\text { IHC+/ } \\
\text { RTqPCR } \\
+\end{array}$ & $\begin{array}{l}\text { IHC+/ } \\
\text { RTqPCR } \\
-\end{array}$ & $\begin{array}{l}\text { IHC-/ } \\
\text { RTqPCR } \\
-\end{array}$ & $\begin{array}{l}\text { IHC-/ } \\
\text { RTqPCR } \\
+\end{array}$ & $\begin{array}{l}\text { Sensitivity } \\
\text { (PPA) }\end{array}$ & $\begin{array}{l}\text { Specificity } \\
\text { (NPA) }\end{array}$ & $\begin{array}{l}\text { Concordance } \\
\text { rate (OPA) }\end{array}$ & $\begin{array}{l}\text { Kappa } \\
\text { Statistic }\end{array}$ \\
\hline $\begin{array}{l}\text { ER/ESR1 } \\
(\mathrm{IHC}+1 \%)\end{array}$ & $\mathrm{IHC}$ & 200 & 142 & 4 & 45 & 9 & $97.26 \%$ & $83.33 \%$ & $93.5 \%$ & 0.830 \\
\hline $\begin{array}{l}\text { ER/ESR1 } \\
(\mathrm{IHC}+10 \%)\end{array}$ & $\mathrm{IHC}$ & 200 & 142 & 4 & 45 & 9 & $97.26 \%$ & $83.33 \%$ & $93.5 \%$ & 0.830 \\
\hline $\begin{array}{l}\text { PR/PGR } \\
(\mathrm{IHC}+1 \%)\end{array}$ & $\mathrm{IHC}$ & 194 & 130 & 5 & 32 & 27 & $96.3 \%$ & $54.24 \%$ & $83.51 \%$ & 0.565 \\
\hline $\begin{array}{l}\text { PR/PGR } \\
(\mathrm{IHC}+10 \%)\end{array}$ & $\mathrm{IHC}$ & 194 & 114 & 1 & 36 & 43 & $99.13 \%$ & $45.57 \%$ & $77.32 \%$ & 0.488 \\
\hline HER2/ERBB2 & $\mathrm{IHC}$ & 175 & 30 & 3 & 136 & 6 & $90.91 \%$ & $95.77 \%$ & $95 \%$ & 0.838 \\
\hline HER2/ERBB2 & FISH & 25 & 7 & 1 & 10 & 7 & $87.50 \%$ & $58.82 \%$ & $68 \%$ & 0.387 \\
\hline HER2/ERBB2 & $\begin{array}{l}\text { IHC/ } \\
\text { FISH }\end{array}$ & 200 & 37 & 3 & 147 & 13 & $92.5 \%$ & $91.88 \%$ & $92 \%$ & 0.771 \\
\hline $\begin{array}{l}\text { HER2/ERBB2 } \\
\text { in ER+ }\end{array}$ & $\begin{array}{l}\mathrm{IHC} / \\
\mathrm{FISH}\end{array}$ & 146 & 11 & 3 & 119 & 13 & $78.57 \%$ & $90.15 \%$ & $89.04 \%$ & 0.521 \\
\hline $\begin{array}{l}\text { HER2/ERBB2 } \\
\text { in ER- }\end{array}$ & $\begin{array}{l}\text { IHC/ } \\
\text { FISH }\end{array}$ & 54 & 26 & 0 & 28 & 0 & $100 \%$ & $100 \%$ & $100 \%$ & 1.000 \\
\hline $\begin{array}{l}\text { KI67/MKI67 } \\
(\mathrm{IHC}+\geq 20 \%)\end{array}$ & $\mathrm{IHC}$ & 184 & 92 & 3 & 32 & 57 & 96.84 & 35.96 & $67.39 \%$ & 0.334 \\
\hline $\begin{array}{l}\text { KI67/MKI67 } \\
(\mathrm{IHC}+>10 \%)\end{array}$ & $\mathrm{IHC}$ & 184 & 94 & 6 & 29 & 55 & $94 \%$ & $34.52 \%$ & $66.85 \%$ & 0.299 \\
\hline $\begin{array}{l}\text { KI67/MKI67 } \\
\text { (excluding } \\
10 \leq \mathrm{HC} \%<20 \\
\text { range) }\end{array}$ & $\mathrm{IHC}$ & 133 & 92 & 3 & 16 & 22 & $96.84 \%$ & $42.11 \%$ & $81.20 \%$ & 0.458 \\
\hline
\end{tabular}

For both ER and PR, we used IHC cutoffs of 1\% as recommended by the ASCO/CAP 2010 ER/PR testing guidelines[29] as well as 10\% as described elsewhere [20,30]. Central IHC and central FISH are used for resolution of IHC HER2 2+ to either FISH-negative or FISH-positive, as recommended by the ASCO-CAP guidelines [28] for HER2 testing. For Ki67 we used IHC cutoff of $\geq 20 \%$ and $>10 \%$ to discriminate "high proliferation rate" from "Iow proliferation rate".

\section{Discussion}

Immunohistochemical assays are the current gold standard for evaluating ER, PR, HER2 and Ki67 status in breast invasive carcinoma. The main advantages of IHC for the assessment of these markers are that it is rapid and simple, it can be performed in most pathology laboratories, and (when compared with other assays) it is relatively inexpensive. However, IHC assay reliability has been questioned because alterations during tissue processing, manipulation and fixation, as well as the antibody clone, internal controls and scoring system used may affect the precision of the results. In addition to that, inter-observer variability in interpretation may play also a role as IHC remains a semiquantitative and non-standardized method[18-20].

The ambiguity encountered in the interpretation of HER2 IHC results especially in cases with HER2 equivocal scores (HER2 IHC =2 + ) may also represent an issue that most laboratories in resource-constrained settings may not be able to overcome as the gold standard would be, in these cases, FISH for quantifying HER-2 gene amplification. Indeed, FISH has the advantage of being a quantitative method and is 
considered as the gold standard method for confirming the HER-2 status, not only to resolve IHC $2+$ cases, but also for all other cases where it has an excellent correlation with the HER2 IHC results[31, 32] Major disadvantages are that FISH is technically complicated to execute, arduous to establish, has a long run time, and is costly, making it not routinely available in all pathology laboratories worldwide. Moreover, another limitation of this method is that it doesn't necessarily reflect target protein expression and counting FISH spots is wearisome and can be biased by tumor heterogeneity $[9,19,20]$.

Nevertheless, these causes of assay variability may explain the differences in ER, PR, HER2, and Ki67 IHC results in breast carcinomas reported previously.

Currently, treatment of invasive breast carcinoma relies essentially upon ER, PR, HER2 and Ki67 status [33, 34] and accuracy of assays is critical. Hence, to overcome limitations of IHC and HER2 FISH, there have been efforts to establish alternative methods to assess the 4 biomarkers of interest as accurately as possible [35]. One of the options is to use RT-qPCR. Reverse transcription quantitative PCR (RT-qPCR) represents a sensitive, efficient, and reliable approach for analyzing RNA. The initial step in RT-PCR is the production of a single-strand complementary DNA copy (CDNA) of the RNA through the action of the retroviral enzyme, reverse transcriptase, to amplify that part of this CDNA by PCR. RT-PCR is used to analyze differential gene expression or cloned cDNAs. RT-PCR is more sensitive and easier to perform than other RNA analysis techniques[36]. Xpert $\circledast$ Breast Cancer STRAT4 is a real-time RT-qPCR semi-quantitative assay with qualitative cut-off values for Estrogen Receptor (ESR1), Progesterone Receptor (PGR), HER2/ERBB2, and the marker of proliferation Ki-67 (MKi67) mRNAs isolated from FFPE tissues. The test is intended to be used with the GeneXpert® System, which automates RNA isolation and purification from FFPE tissue, as well as amplification and detection of target sequences within the cartridge[18, 37].

In this study, our statistical data demonstrated that the Xpert® Breast cancer STRAT4 closed-system RT-qPCR method shows basically a good concordance rate with IHC+HER2 FISH results.

The concordance between Xpert® Breast cancer STRAT4 and HER2 IHC+HER2 FISH has been evaluated in other studies and varies between $91 \%$ and $98 \%$. In the current analysis, our data demonstrate that the Xpert® Breast Cancer STRAT4 assay shows greater than $91 \%$ concordance with HER2 IHC+HER2 FISH, suggesting that our results are generally concordant with the previous studies[16-20,32, 38].

For both ESR1/ER and ERBB2/HER2, data suggested almost perfect agreement between Xpert® Breast Cancer STRAT4 and central IHC ( $\mathrm{K}$ "ER" $=0.830 ; \mathrm{k}$ "HER2"= 0.838), with nearly all of the discordant cases with quantitative dCt values close to the $E S R 1$ and $E R B B 2 \mathrm{~d} C_{\mathrm{t}}$ cutoffs, respectively. Our findings are in good agreement with previously reported results: we found overall concordance of $93.50 \%$ and $95 \%$ for ER and HER2 respectively, and previously published papers have reported values of $97-98 \%$ for ER and of $93-97 \%$ for HER2[16-18, 20].

The results showed a moderate Kappa correlation agreement for PGR/PR (using PR IHC+ 1\%) and MKi67/Ki67 (excluding equivocal cases) between both assays ( $\mathrm{K}$ "PR" $=0.565 ; \mathrm{K}$ "Ki67"= 0.458). Xpert $\circledast$ Breast Cancer STRAT4, however, demonstrated a significant overall concordance with IHC for PGR (83.5\%) and MKi67 (81\%). The concordance rates observed in other studies vary from $81-92 \%$ for PR and from $78-89 \%$ for Ki67, in accordance with agreement percentages obtained in our analysis[16-18, 20, 38].

Discordance between assay methods can be attributed to several factors, including the tissue fixation, antibody clone used in IHC, and scoring methods used. Preanalytical factors are essential to monitor and a quality assessment scheme should be put in place in any laboratory routinely performing the assessment of the 4 biomarkers [39]. Particular attention should be paid to the impact of sample handling, time of fixation, duration of tissue fixation, antibody selection, control samples and interpretation of assay on Xpert ${ }^{\circledR}$ Breast Cancer STRAT4 results $[8,10,11,14]$. In spite of the systematic practice of immunohistochemistry methods, procedural inconsistency remains elevated in clinical settings, leading to interlaboratory and intralaboratory variations and to high false-negative (for ER and PR) and false-positive (for HER2)[15]. This inconsistency emphasizes the importance of a standardized retrieval method in the performance of reliable IHC and/ or FISH for the four markers routinely screened in breast cancer diagnosis.

Xpert@ Breast Cancer STRAT4 has already been shown to have good agreement with automated semi-quantitative IHC[16-18, 21, 22, 24]. ESR1 and ERBB2 assessments have the highest pertinence in all studies. Comparison between the Xpert® Breast Cancer STRAT4 PGR status and the PR IHC status resulted in more discrepancies. This discrepancy between the two methods could be explained by the fact that total mRNA does not necessarily reflect the total protein and vice versa [40]. Denkert et al. have demonstrated that Ki67 IHC results are greatly variable. For this biomarker, a significant variability in concordance rate has been noted, although this is not unforeseen given the challenges associated with $\mathrm{Ki} 67 \mathrm{IHC}$ evaluation[18].

Considering our results, as well as other similar results found in other published papers, the Xpert® Breast Cancer STRAT4 assay could be a potential solution to overcome IHC/FISH limitations and may help facilitate access to invasive breast cancer testing in low resource 
countries. It is a sensitive method that can replace IHC and FISH in remote areas where IHC cannot be performed, because it is a nonoperator -dependent technique and does not require an equipped molecular laboratory[9, 11, 16-20, 38].

\section{Conclusion}

Determination of hormone receptors (ER/PR), HER2 and Ki67 status by immunohistochemistry (and in situ hybridization for HER2 IHC 2+ cases) is part of the standard management of invasive breast cancer. There are questions related to technical issues with the standard tests used and all international recommendations insist on improving the quality of tissue samples analyzed (pre-analytical phase), analytical techniques and interpretation of results to ensure quality immunohistochemistry and molecular biology tests. The new RT-qPCR assay « Xpert ${ }^{\circledR}$ Breast Cancer STRAT4 » gave overall very promising results and a remarkable agreement with the reference techniques (IHC and FISH). Molecular diagnostics have become more and more essential in the diagnosis and a good management of cancer in general, and further studies of Xpert® Breast Cancer STRAT4 are needed with a larger sample size to support and confirm the results already published.

\section{Abbreviations}

\section{List of abbreviations}

HER2 or ERBB2: Human Epidermal Growth Factor Receptor 2

KI67 or MKi67: marker of proliferation Ki67

ESMO: European Society for Medical Oncology

ER or ESR1: estrogen receptor

PR or PGR: progesterone receptor

IHC: immunohistochemistry

FFPE: formalin-fixed paraffin-embedded

FISH: Fluorescence in situ hybridization

CYFIP1: Cytoplasmic FMR1-interacting protein 1

GX: GeneXpert ${ }^{\circledR}$

RT-PCR : real-time reverse transcriptase, polymerase chain reaction

RT-qPCR : Reverse transcription quantitative PCR

SOC : standard of care

ASCO/CAP : American Society of Clinical Oncology and the College of American Pathologists

K : Cohen's k coefficient

dCt: delta Ct cut-off

PPA: Positive Percent Agreement

NPA : Negative Percent Agreement

PPV : Positive predictive value

NPV : Negative predictive value

CEP17: centromeric region of chromosome 17

cDNA: Complementary DNA

mRNA: Messenger RNA 


\section{Declarations}

\section{Ethics approval and consent to participate}

All experiments were performed retrospectively and in accordance with the Moroccan Bioethics Law 28-13, and after approval by the Casablanca biomedical research ethics committee (CERBC).

\section{Consent for publication}

Not applicable

\section{Availability of data and materials}

The datasets used and/or analysed during the current study are available from the corresponding author on reasonable request.

\section{Competing interests}

J.W. was an employee of Cepheid at the time of this study.

The funders had no role in the selection of samples used in the study, in the collection of data from participating sites, the interpretation of the final data analyses in the study, or the decision to publish the results.

The corresponding authors had sole final responsibility for preparing the original draft manuscript, finalizing data analyses, or interpretation of data, in the writing of the manuscript, and in the decision to publish the results.

The other authors declare that they have no competing interests.

\section{Funding}

This study was funded by Cepheid®. All Xpert® Breast Cancer STRAT4 (CE-IVD) kits were provided by Cepheid. Cepheid was not involved in sample selection, nor the final data analysis.

\section{Authors' contributions}

RE Realised the technical part, analyzed and interpreted the data and was a major contributor in writing the manuscript. AN Study monitoring and corrected the manuscript. AK selected samples with the pathologist and Contributed to the technical analysis. YZ contributed to the statistical study. MO corrected the manuscript. JW corrected the manuscript. MK the study supervisor, selected eligible cases for the study, reviewed the H\&E stained slide, Interpreted the data and Corrected the manuscript. All authors read and approved the final manuscript.

\section{Acknowledgments}

The authors would like to thank all the collaborators who ensured the good progress of this study.

\section{References}

1. Bray F, Ferlay J, Soerjomataram I, Siegel RL, Torre LA, Jemal A. Global cancer statistics 2018: GLOBOCAN estimates of incidence and mortality worldwide for 36 cancers in 185 countries. CA Cancer J Clin. Nov 2018; 68(6):394-424.

2. Odonate Therapeutics Announces Initiation of CONTESSA, a Phase 3 Study of Tesetaxel in Patients with Locally Advanced or Metastatic Breast Cancer [Internet]. Accessed 2021 Jan 14 https://www.firstwordpharma.com/node/1533455

3. International Agency for Research on Cancer. 504-morocco-fact-sheets.pdf. Updated 2020 Accessed Jan 2021. https://gco.iarc.fr/today/data/factsheets/populations/504-morocco-fact-sheets.pdf 
4. DeSantis C, Ma J, Bryan L, Jemal A. Breast cancer statistics, 2013: Breast Cancer Statistics, 2013. CA A Cancer Journal for Clinicians. Jan 2014;64(1):52-62.

5. Allegra JC, Lippman ME, Thompson EB, Simon R, Barlock A, Green L, et al. Estrogen receptor status: an important variable in predicting response to endocrine therapy in metastatic breast cancer. European Journal of Cancer (1965). Mar 1980;16(3):323-31.

6. Early Breast Cancer Trialists' Collaborative Group (EBCTCG). Relevance of breast cancer hormone receptors and other factors to the efficacy of adjuvant tamoxifen: patient-level meta-analysis of randomised trials. The Lancet. Aug 2011;378(9793):771-84.

7. Osborne CK. Tamoxifen in the Treatment of Breast Cancer. Wood AJJ, N Engl J Med. 1998 Nov 26;339(22):1609-18.

8. Bardou V-J, Arpino G, Elledge RM, Osborne CK, Clark GM. Progesterone Receptor Status Significantly Improves Outcome Prediction Over Estrogen Receptor Status Alone for Adjuvant Endocrine Therapy in Two Large Breast Cancer Databases. JCO. 2003 May 15;21(10):1973-9.

9. Press MF, Slamon DJ, Flom KJ, Park J, Zhou J-Y, Bernstein L. Evaluation of HER-2/ neu Gene Amplification and Overexpression: Comparison of Frequently Used Assay Methods in a Molecularly Characterized Cohort of Breast Cancer Specimens. JCO. 2002 Jul 15;20(14):3095-105.

10. Cuzick J, Dowsett M, Pineda S, Wale C, Salter J, Quinn E, et al. Prognostic Value of a Combined Estrogen Receptor, Progesterone Receptor, Ki-67, and Human Epidermal Growth Factor Receptor 2 Immunohistochemical Score and Comparison With the Genomic Health Recurrence Score in Early Breast Cancer. JCO. 2011 Nov 10;29(32):4273-8.

11. Dowsett M, Nielsen TO, A'Hern R, Bartlett J, Coombes RC, Cuzick J, et al. Assessment of Ki67 in Breast Cancer: Recommendations from the International Ki67 in Breast Cancer Working Group. JNCI Journal of the National Cancer Institute. 2011 Nov 16;103(22):1656-64.

12. Allison KH, Hammond MEH, Dowsett M, McKernin SE, Carey LA, Fitzgibbons PL, et al. Estrogen and Progesterone Receptor Testing in Breast Cancer: ASCO/CAP Guideline Update. Journal of Clinical Oncology. 2020 Jan 13:23.

13. Xu B, Shen J, Guo W, Zhao W, Zhuang Y, Wang L. Impact of the 2018 ASCO/CAP HER2 guidelines update for HER2 testing by FISH in breast cancer. Pathology - Research and Practice. 2019 Feb 1;215(2):251-5.

14. Cardoso F, Kyriakides S, Ohno S, Penault-Llorca F, Poortmans P, Rubio IT, et al. Early breast cancer: ESMO Clinical Practice Guidelines for diagnosis, treatment and follow-upt. Annals of Oncology. 2019 Aug 1;30(8):1194-220.

15. Roepman P, Horlings HM, Krijgsman O, Kok M, Bueno-de-Mesquita JM, Bender R, et al. Microarray-based determination of estrogen receptor, progesterone receptor, and HER2 receptor status in breast cancer. Clin Cancer Res. 2009 Nov 15;15(22):7003-11.

16. Wong E, Wu N, Acca B, Dias H, Shao S, Wong W, Weidler J, Bates M, HO K, Chu V, Rizo A, Malek M, David K, Quigley NB, Beqaj SS, Davenport S, Press M. GeneXpert ${ }^{\circledR}$ Breast Cancer STRAT4 assay Demonstrates High Concordance of ESR1, PgR, HER2, and Ki67 with Central IHC and FISH testing in FFPE Breast Tumor Tissues. 15th St. Gallen International Breast Cancer Conference, Vienna (2017). Abstract Poster P077.

17. Wong W, Ho KE, Wu N, Chu VC, Lalli P, Longshore JW, et al. Highly reproducible decentralized gene expression analysis of ESR1, PGR, ERBB2 and MKi67 on an automated, standardized molecular diagnostics platform, GeneXpert®. Abstract P1-03-09: 2016 San Antonio Breast Cancer Symposium; December 6-10, 2016. San Antonio, Texas.

18. Denkert C, Link T, Jank P, Just M, Hanusch C, Brasch F, et al. Comparison of an automated cartridge-based system for mRNA assessment with central immunohistochemistry in the neoadjuvant GeparX trial. JC0. 2019 May 20;37(15_suppl):3075-3075.

19. Wasserman BE, Carvajal-Hausdorf DE, Ho K, Wong W, Wu N, Chu VC, et al. High concordance of a closed-system, RT-qPCR breast cancer assay for HER2 mRNA, compared to clinically determined immunohistochemistry, fluorescence in situ hybridization, and quantitative immunofluorescence. Lab Invest. Dec 2017;97(12):1521-6.

20. Wu NC, Wong W, Ho KE, Chu VC, Rizo A, Davenport S, et al. Comparison of central laboratory assessments of ER, PR, HER2, and Ki67 by IHC/FISH and the corresponding mRNAs (ESR1, PGR, ERBB2, and MKi67) by RT-qPCR on an automated, broadly deployed diagnostic platform. Breast Cancer Res Treat. Nov 2018;172(2):327-38.

21. Janeva S, Parris TZ, Nasic S, De Lara S, Larsson K, Audisio RA, et al. Comparison of breast cancer surrogate subtyping using a closedsystem RT-qPCR breast cancer assay and immunohistochemistry on 100 core needle biopsies with matching surgical specimens. BMC Cancer. Dec 2021;21(1):439.

22. Filipits M, Rudas M, Singer CF, Fitzal F, Bago-Horvath Z, Greil R, et al. ESR1, PGR, ERBB2, and MKi67 mRNA expression in postmenopausal women with hormone receptor-positive early breast cancer: results from ABCSG Trial 6. ESMO Open. Aug 2021;6(4):100228.

23. Erber R, Hartmann A, Fasching PA, Ruebner M, Stöhr R, Beckmann MW, et al. Reproducibility of mRNA-Based Testing of ESR1, PGR, ERBB2, and MKI67 Expression in Invasive Breast Cancer-A Europe-Wide External Quality Assessment. Cancers. 2021 Sep $21 ; 13(18): 4718$. 
24. Mugabe M, Ho KE, Ruhangaza D, Milner D, Rugwizangoga B, Chu VC, et al. Use of the Xpert® Breast Cancer STRAT4 for Biomarker Evaluation in Tissue Processed in a Developing Country. American Journal of Clinical Pathology. 2021 May 29 ; aqab016.

25. Sams SB. American Society of Clinical Oncology/College of American Pathologists Guideline Recommendations for Immunohistochemical Testing of Estrogen and Progesterone Receptors in Breast Cancer (Unabridged Version). Yearbook of Pathology and Laboratory Medicine. Jan 2011; 2011:25-6.

26. Wolff AC, Hammond MEH, Schwartz JN, Hagerty KL, Allred DC, Cote RJ, et al. American Society of Clinical Oncology/College of American Pathologists Guideline Recommendations for Human Epidermal Growth Factor Receptor 2 Testing in Breast Cancer. Journal of Clinical Oncology. 2007 Jan 1;26(1):118-45.

27.27

28. Gordian-Arroyo AM, Zynger DL, Tozbikian GH. Impact of the 2018 ASCO/CAP HER2 Guideline Focused Update. American Journal of Clinical Pathology. 5 juin 2019;152(1):17-26.

29. Hammond MEH, Hayes DF, Wolff AC, Mangu PB, Temin S. American Society of Clinical Oncology/College of American Pathologists Guideline Recommendations for Immunohistochemical Testing of Estrogen and Progesterone Receptors in Breast Cancer. JOP. Jul 2010;6(4):195-7.

30. Tang P, Tse GM. Immunohistochemical Surrogates for Molecular Classification of Breast Carcinoma: A 2015 Update. Archives of Pathology \& Laboratory Medicine. 2016 Aug 1;140(8):806-14.

31. Callata-Carhuapoma HR, Sotelo Lezama M, Cabezas S, Garcia Saenz JA, Moreno F, Serrano G, et al. Concordance between immunohistochemistry (IHC) and fluorescence in situ hybridization (FISH) for HER2 determination and correlation with clinical and pathological data. JCO. 2015 May 20;33(15_suppl):e11616-e11616.

32. Middleton LP, Price KM, Puig P, Heydon LJ, Tarco E, Sneige N, et al. Implementation of American Society of Clinical Oncology/College of American Pathologists HER2 Guideline Recommendations in a tertiary care facility increases HER2 immunohistochemistry and fluorescence in situ hybridization concordance and decreases the number of inconclusive cases. Arch Pathol Lab Med. May 2009;133(5):775-80.

33. Cardoso F, Paluch-Shimon S, Senkus E, Curigliano G, Aapro MS, André F, et al. 5th ESO-ESMO international consensus guidelines for advanced breast cancer (ABC 5). Ann Oncol. Dec 2020;31(12):1623-49.

34. Cardoso F, Kyriakides S, Ohno S, Penault-Llorca F, Poortmans P, Rubio IT, et al. Early breast cancer: ESMO Clinical Practice Guidelines for diagnosis, treatment and follow-upt. Ann Oncol. 2019 Aug 1;30(8):1194-220.

35. El Hadi H, Abdellaoui-Maane I, Kottwitz D, El Amrani M, Bouchoutrouch N, Qmichou Z, et al. Development and evaluation of a novel RTqPCR based test for the quantification of HER2 gene expression in breast cancer. Gene. 2017 Mar 20; 605:114-22.

36. Freeman WM, Walker SJ, Vrana KE. Quantitative RT-PCR: Pitfalls and Potential. BioTechniques. Jan 1999;26(1):112-25.

37. Denkert C, Jank P, Link T. Expression of ER, PR, HER2 and Ki67 in the neoadjuvant GeparX trial: Comparison of central immunohistochemistry with an automated cartridge-based system for mRNA assessment (on behalf of the GBG neoadjuvant and translational subboard). Annals of Oncology. 2019 May 1;30: iii8-9.

38. Filipits M. mRNA expression of ER, PR, HER2 and Ki67 are concordant to central inc and predict clinical outcome: A validation study from the ABCSG-6 biomarker cohort. Annals of Oncology. Oct 2018. Volume 29 | Supplement 8

39. Fitzgibbons PL, Murphy DA, Hammond MEH, Allred DC, Valenstein PN. Recommendations for validating estrogen and progesterone receptor immunohistochemistry assays. Arch Pathol Lab Med. Jun 2010;134(6):930-5.

40. Gupta S, Neumeister V, McGuire J, Song YS, Acs B, Ho K, et al. Quantitative assessments and clinical outcomes in HER2 equivocal 2018 ASCO/CAP ISH group 4 breast cancer. npj Breast Cancer. 2019 Aug 29;5(1):1-8.

\section{Figures}




\section{ESR1 dCt vs. IHC\% Staining}

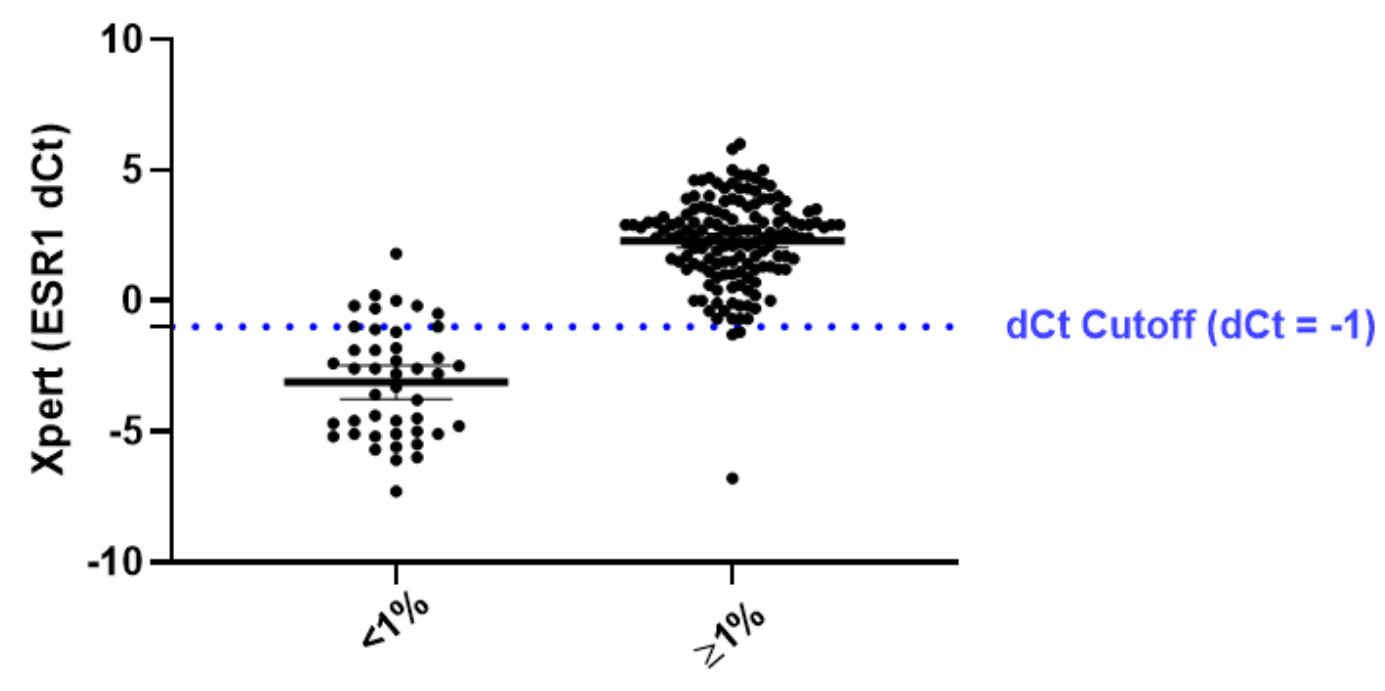

IHC (\% ER-positive cells)

Figure 1

Comparison of estrogen receptor status determined by RT-qPCR and Immunohistochemistry. Graph of mRNA expression ESR1 dCt determinated with Xpert® Breast Cancer STRAT4 test* by ER IHC result categorized as negative (<1\%), or positive ( $\geq 1 \%)$.

\section{PGR dCt vs. IHC\% Staining}

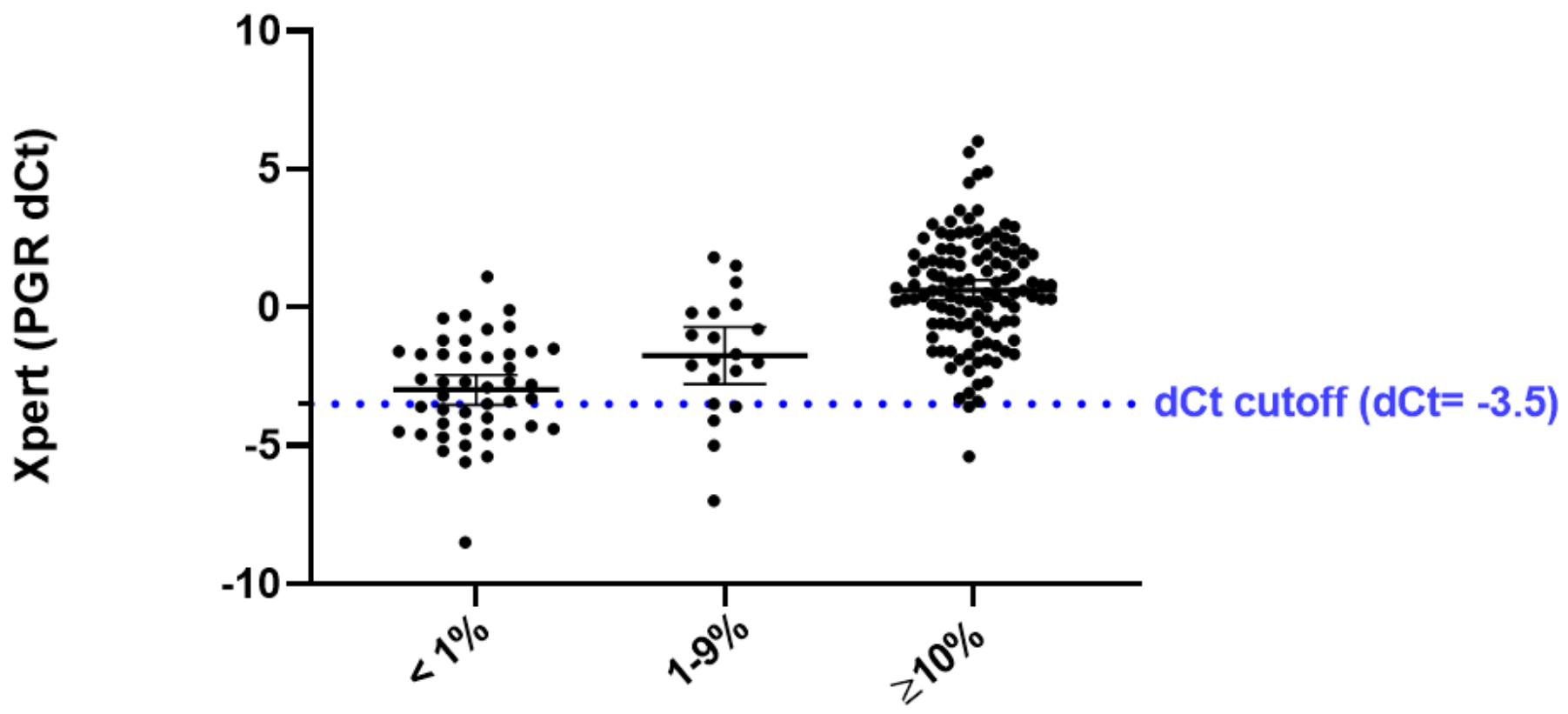

IHC (\% PR-positive cells)

Figure 2 


\section{ERBB2 dCt vs. IHC}

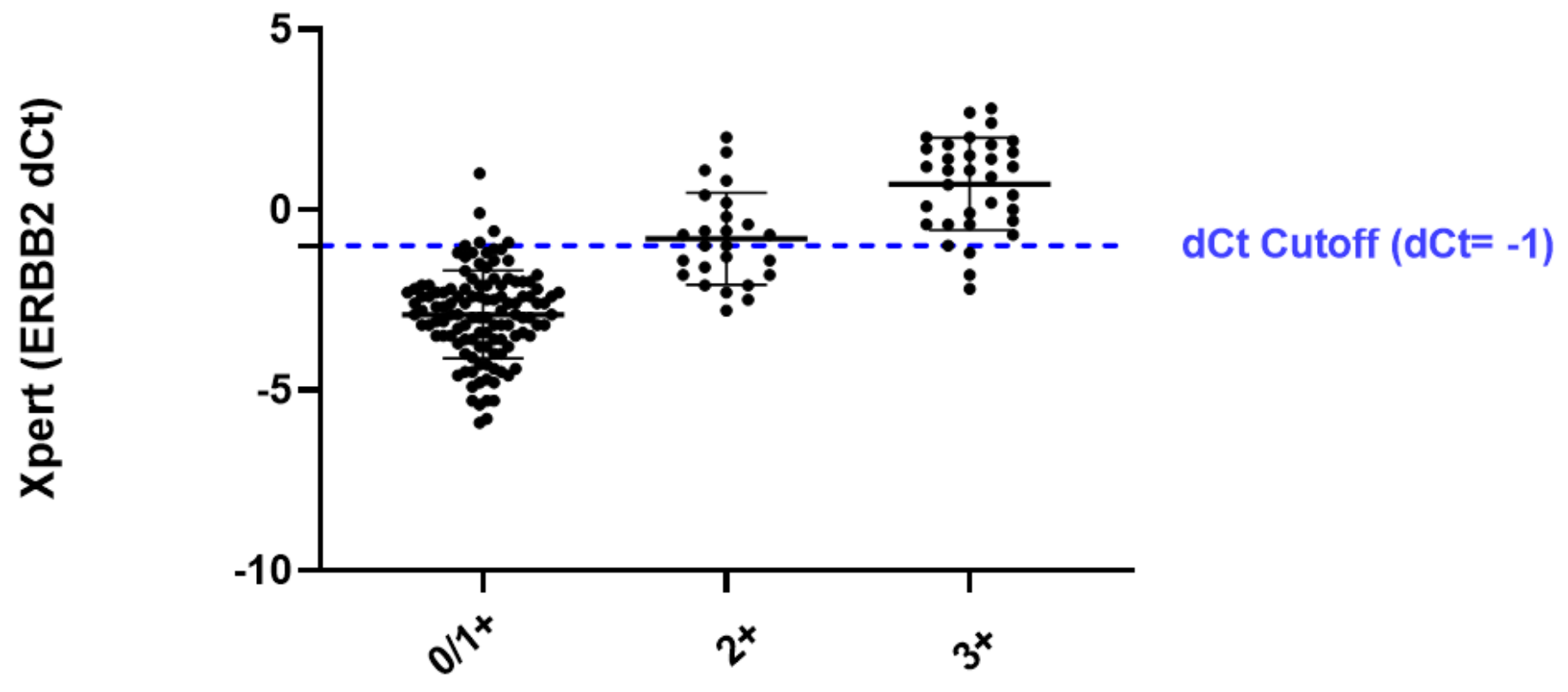

Her2 IHC

Figure 3

Comparison of HER2/ERBB2 determined by RT-qPCR and immunohistochemistry. Graph of mRNA expression ERBB2 dCt determined with Xpert ${ }^{\circledR}$ Breast Cancer STRAT4 test by HER2 IHC result categorized as negative $(0-1+)$, equivocal $(2+)$, or positive (3+).

\section{ERBB2 dCt vs. FISH}

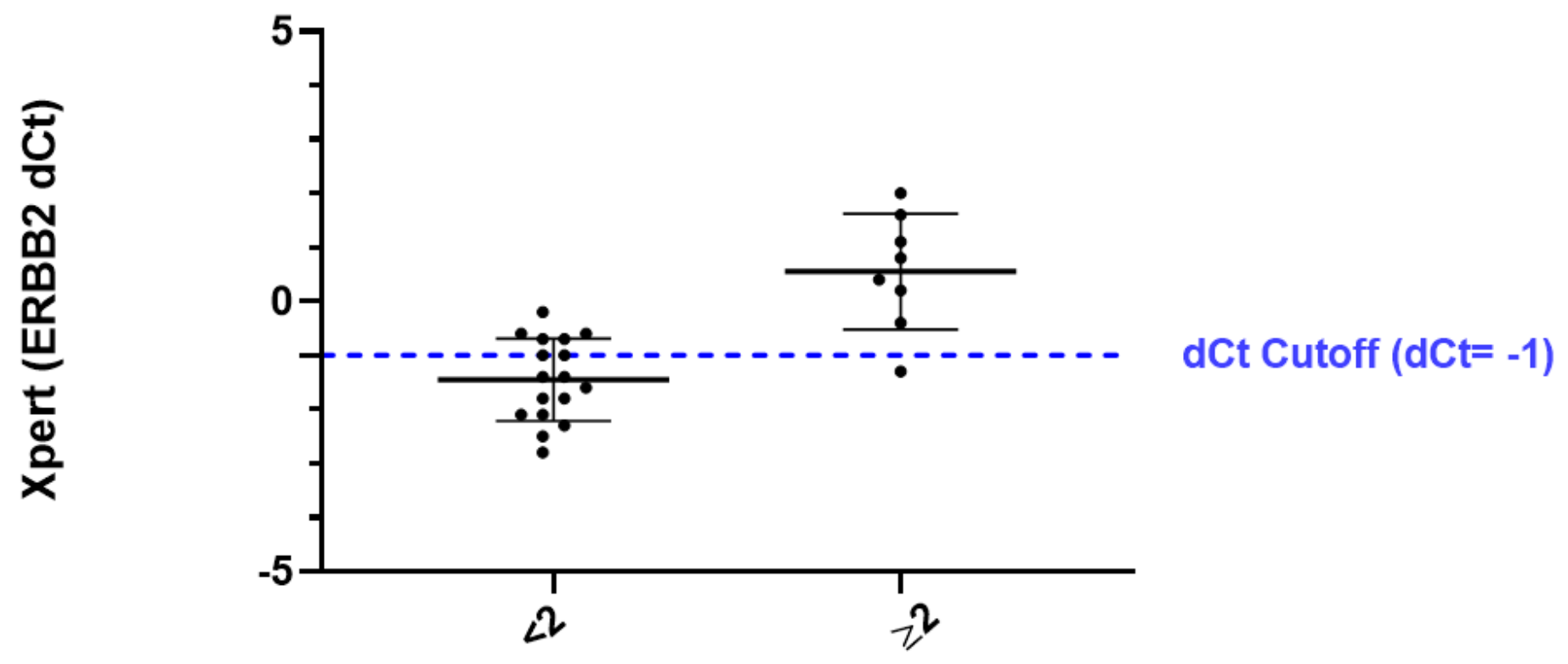

Her2/CEP17 Ratio

Figure 4 

positive « Amplified» ( $\geq 2)$.

\section{ERBB2 dCt vs. IHC (2+ with FISH Results)}

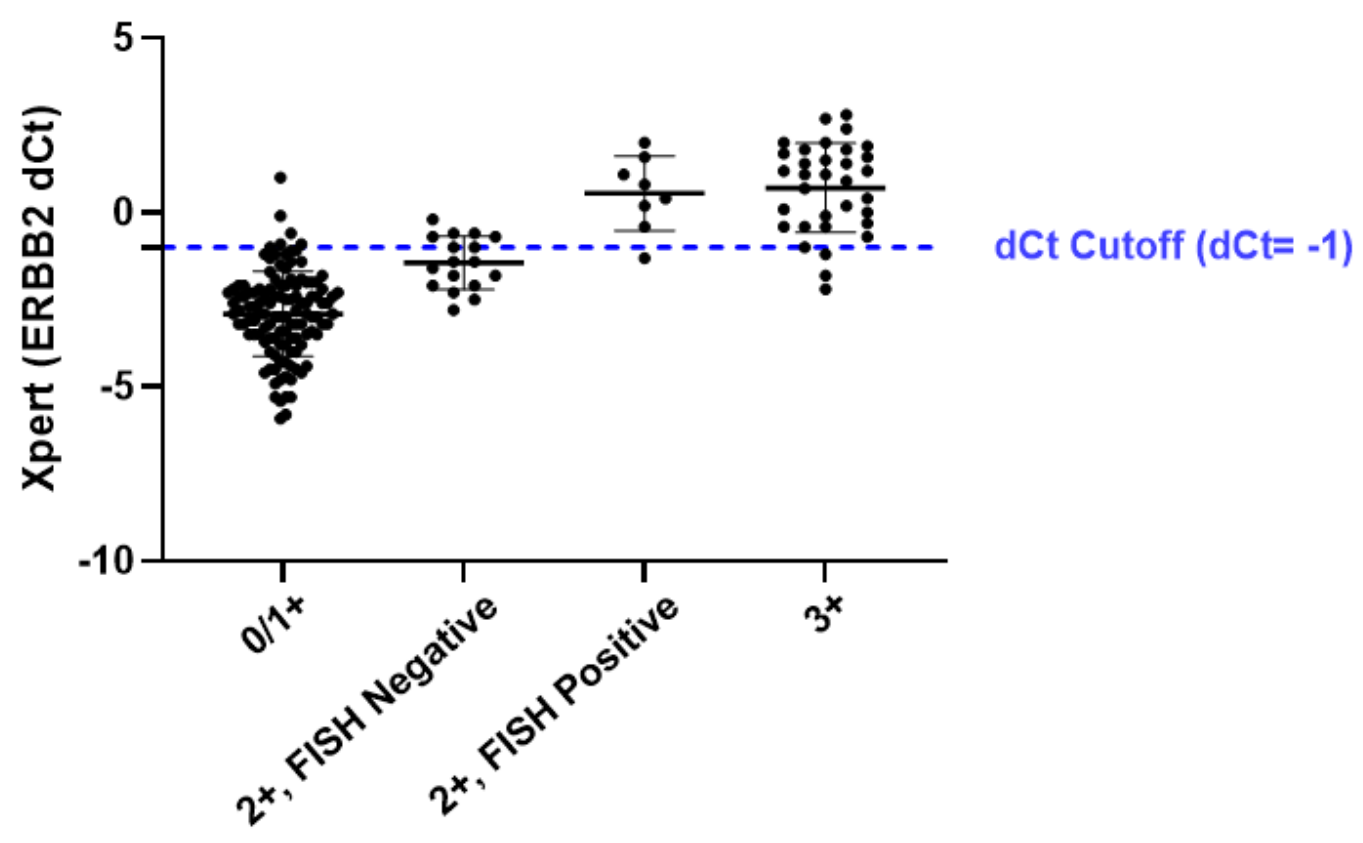

IHC (Her2, 2+ with FISH Results)

\section{Figure 5}

Comparison of HER2/ERBB2 determined by either RT-qPCR or by immunohistochemistry with or without FISH assessment of IHC2+. Graph of mRNAexpression ERBB2 dCt determined with Xpert® Breast Cancer STRAT4 test by IHC/FISH Her2 results including all sample size. 


\section{MKI67 dCt vs. IHC\% Staining}

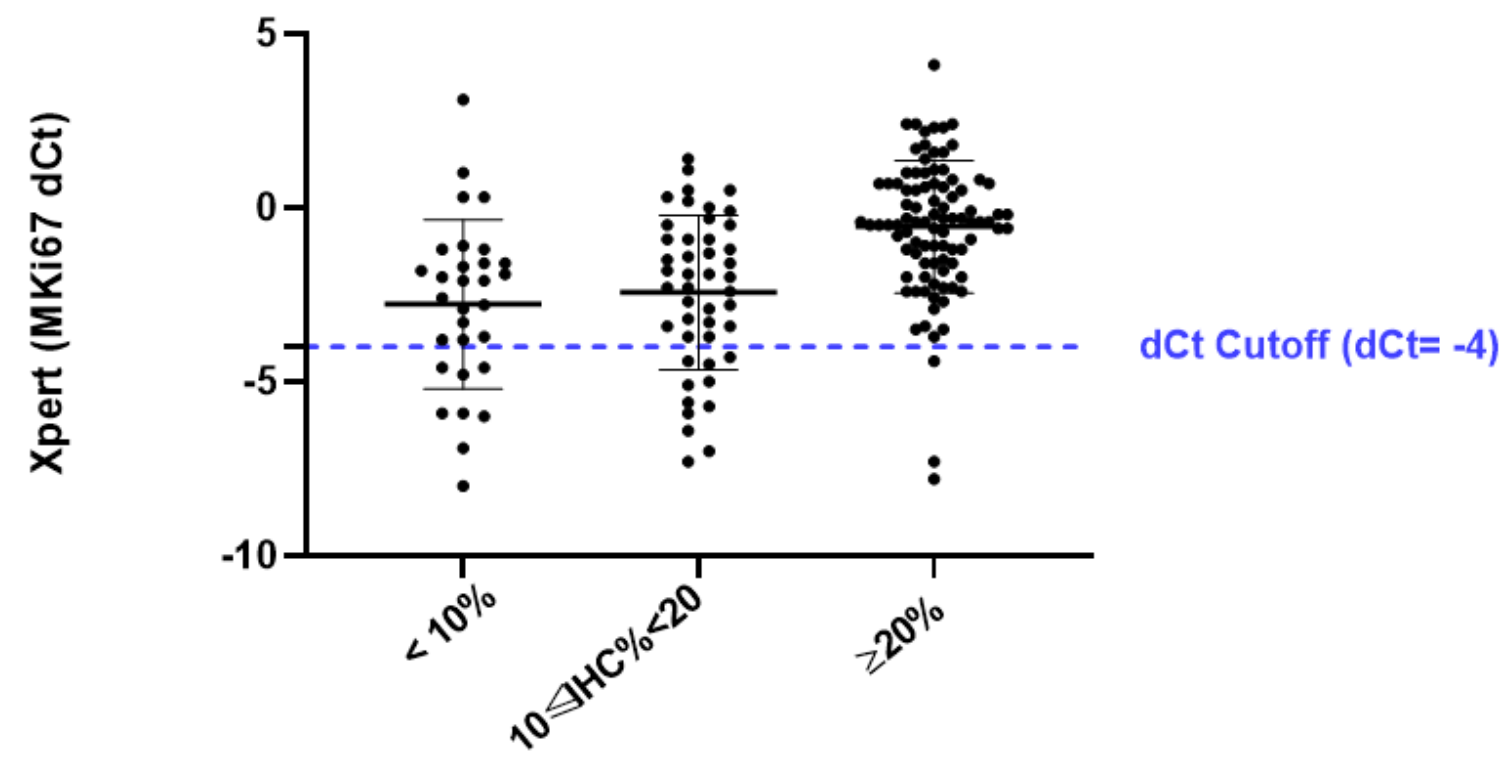

IHC (\%Ki67- Positive cells)

Figure 6

Comparison of Ki67 proliferation rate determined by either RT-qPCR or immunohistochemistry. Graph of Xpert ${ }^{\circledR}$ Breast Cancer STRAT4 MKi67 dCt values by $\mathrm{Ki} 67 \mathrm{IHC} \%$ staining where the IHC low proliferation rate cutoff is defined as $<10 \%$, IHC high proliferation rate cutoff is defined as $\geq 20 \%$ and the intermediate proliferation rate (equivocal range) is defined as $10 \% \leq \mathrm{IHC} \%<20 \%$. 